Nouvelles perspectives en sciences sociales

Revue internationale de systémique complexe et d'études relationnelles

\title{
Prédiction et prévision en histoire économique : les succès suisses et scandinaves étaient-ils prévisibles?
}

\section{Jean-Marc Olivier}

Volume 6, numéro 2, juin 2011

Sur le thème : «Les économistes dans la Cité »

URI : https://id.erudit.org/iderudit/1005771ar

DOI : https://doi.org/10.7202/1005771ar

Aller au sommaire du numéro

Éditeur(s)

Prise de parole

ISSN

1712-8307 (imprimé)

1918-7475 (numérique)

Découvrir la revue

Citer cet article

Olivier, J.-M. (2011). Prédiction et prévision en histoire économique : les succès suisses et scandinaves étaient-ils prévisibles? Nouvelles perspectives en sciences sociales, 6(2), 87-96. https://doi.org/10.7202/1005771ar

\section{Résumé de l'article}

L'historien de l'économie dispose de multiples outils pour analyser les réussites économiques. Il parvient le plus souvent à les rendre logiques et évidentes, mais en ayant la chance de travailler sur des évènements passés qui ne peuvent pas contredire son raisonnement. Toutefois, l'analyse des plus beaux succès - suisses et scandinaves - nuance les modèles les plus couramment admis : comme l'exploitation de ressources naturelles abondantes, la révolution industrielle libérale à l'anglaise ou le triomphe de la grande usine. En effet, les développements suisse, suédois et norvégien offrent de belles pistes de réflexion car ils reposent davantage sur les PME, sur des politiques volontaristes et sur une maîtrise ancienne des statistiques. 


\title{
Prédiction et prévision en histoire économique : les succès suisses et scandinaves étaient-ils prévisibles?
}

\author{
Jean-Marc Olivier \\ Université de Toulouse - Le Mirail
}

Thistorien de l'économie, qui est souvent un économiste dans prévisionnistes : il doit expliquer pourquoi des événements passés - crises, dépressions ou décollages - se sont produits. Or, d'après l'une des lois de Murphy (loi de la logique absurde) : "Tout événement, une fois qu'il s'est produit, peut être décrit comme inévitable par un historien compétent ». Ce paradigme a inspiré certains ouvrages anciens, tentons de le nuancer en revisitant le cheminement de quelques pays qui n’auraient pas dû se développer.

\section{Richesse et pauvreté imprévisibles des nations}

Reprenons les arguments les plus utilisés dans les manuels d'histoire économique du $\mathrm{XX}^{\mathrm{e}}$ siècle et appliquons-les au devenir de deux États dont la taille et la population étaient voisines au début du XIX ${ }^{e}$ siècle, à l'aube de l'ère des révolutions industrielles. Le premier est enclavé sans débouché sur la mer, il a un relief très accidenté, des fleuves rarement navigables, des sols pauvres, aucun minerai ou presque. Sa population parle trois langues 
différentes et appartient à deux religions antagonistes au bord de l'affrontement au début du XIX ${ }^{e}$ siècle. Enfin, son climat est rude pendant une bonne partie de l'année, coupant les voies de communication. L'autre pays est situé au bord d'une mer chaude, il bénéficie d'un climat favorisant de multiples activités agricoles et regorge de richesses naturelles (fer, bois, énergie hydraulique, or, diamant...). La majorité de sa population pratique la même religion et son élite parle anglais.

Evidemment, la seconde nation semble disposer de tous les atouts favorables à un développement économique brillant au fil des $\mathrm{XIX}^{e}$ et $\mathrm{XX}^{e}$ siècles, par opposition à la première qui n'en possède aucun selon les critères de l'histoire économique imprégnée de déterminisme géographique comme on la pratiquait souvent pendant le $\mathrm{XX}^{\mathrm{e}}$ siècle. Observons maintenant la situation de ces deux espaces en 1975 quand les chiffres de l'indice de développement humain du PNUD sont publiés : le fameux IDH qui combine PIB/h (PPA), espérance de vie, taux d'alphabétisation des adultes et scolarisation des enfants ${ }^{1}$. Le premier pays, accumulant les handicaps selon l'historiographie traditionnelle, arrive pourtant en tête avec un IDH de 0,88: il s'agit de la Suisse $^{2}$. Quant au second, doté de tous les atouts théoriques lui promettant l'opulence, il se classe dernier, avec un IDH de 0,20 : c'est la Sierra Léone, petit État de l'Afrique de l'ouest, encore en paix à cette date. Ces chiffres et ces classements correspondent à une certaine réalité que j’ai pu vérifier dans les faits. En effet, d'origine suisse par ma mère et ayant vécu pendant de longues années en Franche-Comté, je connais bien la Confédération helvétique et l'envie que suscite l'aisance de ses habitants de l'autre côté de la frontière. Plus tard, un autre hasard de la vie m’a permis de découvrir en profondeur la Sierra Léone où j'ai

$1 \quad$ Claude Grasland, " 25 ans de développement humain », dans Images économiques du monde, Paris, Armand Colin, 2005, p. 43-54. L'IDH a été créé en 1990 par le Programme des Nations unies pour le développement (PNUD) sous l'impulsion de Mahbub ul Haq, mais les indices des années antérieures ont été calculés rétroactivement jusqu'en 1975.

2 Jean-François Bergier, Histoire économique de la Suisse, Paris, Armand Colin, 1984. 
vécu de 1986 à 1988 comme volontaire du service national actif (VSNA). Affecté à l'école normale de Milton Margai Teacher's College, près de Freetown, j'ai sillonné l'ensemble du pays afin de rendre visite à mes étudiants en stage dans différentes écoles. Constater de près des écarts considérables de développement constitue une expérience extraordinaire qui relativise l'idée d'un rattrapage entre pays à salaires très bas et pays à salaires très élevés par simple délocalisation des industries.

La Suisse, avec ses hauts salaires, continue d'ailleurs de déjouer les prédictions économiques quand à partir des années 1970 elle doit faire face à la menace des montres à quartz commercialisées par l'entreprise japonaise Seiko. Les observateurs pensent alors que l'horlogerie helvétique fondamentalement mécanique va disparaître. Or, rien de tel ne se produit, aujourd'hui, en chiffre d'affaires, l'horlogerie suisse demeure la première mondiale. Mieux encore, non contente d'occuper sans partage le créneau très profitable du luxe, elle se targue d'être la plus innovante dans le domaine des montres à quartz grâce au groupe Swatch de Nicolas Hayek, ce dernier devenant l'exemple type de l'entrepreneur selon Schumpeter pour les années 1980. Au cœur de cette phase de destruction créatrice, Nicolas Hayek fusionne en 1983 deux entreprises horlogères helvétiques moribondes : SSIH (marques Omega, Tissot...) et ASUAG (marques Longines, Rado et ETA pour les composants...). En 1986, il crée la Société suisse de microélectronique et d'horlogerie (SMH), rebaptisée en 1998 "Swatch groupe ». Pendant cette période, Nicolas Hayek imagine le concept de SwatchMobile qui devient plus tard la Smart. Aujourd'hui Swatch est le premier groupe mondial d'horlogerie avec un quart du chiffre d'affaires planétaire dans ce domaine. D'autres marques de luxe séculaires font désormais partie de son giron comme Breguet, Jaquet Droz ou Blancpain. Cette réussite industrielle s'effectue sans charbon ni minerai de fer. De plus, les entreprises horlogères les plus performantes techniquement sont implantées dans l'une des régions les plus enclavées d'Europe occidentale : la vallée suisse de Joux, toute proche de celle de Mouthe célèbre pour ses records de froid. 
Ainsi, au cœur de la chaîne jurassienne, entre Genève et Besançon, Blancpain (Swatch groupe) et Jaeger-LeCoultre (groupe Richemont) élaborent dans de petites usines les montres les plus complexes du monde.

La Suisse contredit donc les beaux enchaînements logiques d'un essor productif rationnel fondé sur le factory system cher aux historiens de l'économie de l'après Seconde Guerre mondiale. Il en résulte une incapacité à trouver un modèle de développement pour les deux siècles de révolution industrielle, malgré les efforts de conceptualisation menés depuis Walt Whitman Rostow et son ouvrage The Stages of Economic Growth ${ }^{3}$. Ceci s'explique par une trop grande polarisation sur le modèle anglais de la première révolution industrielle. Faut-il pour autant renoncer à toute tentative de modélisation et donc de prévision?

\section{Diversité des itinéraires de développement}

De nombreux pays ont choisi d'autres voies que le modèle anglais, en particulier la Suisse et les pays scandinaves dont les performances exprimées par habitant accaparent les premières places de tous les classements depuis un demi siècle. Ainsi, dès 1960, le PNB/h suédois s'établit à 2000 dollars de l'époque, soit près de $50 \%$ de plus que celui de la France; en 1970, toujours en dollars courants, il atteint le seuil des 4000 , c'est-à-dire pratiquement le double du $\mathrm{PNB} / \mathrm{h}$ des Britanniques. Les premiers classements fondés sur l'IDH placent également en tête les pays du nord depuis les années 1970 jusqu'à aujourd'hui. Ainsi, la Norvège classée première en 2010, était déjà quatrième en 1980 , quant à la Suède, elle se maintient autour du huitième rang qu'elle occupait déjà en $1980^{4}$. Mieux encore, les indicateurs sexo-spécifiques qui prennent en compte les inégalités hommes/ femmes induisent un meilleur classement des pays scandinaves. Cette volonté des pays nordiques, peu peuplés, de mobiliser toutes leurs potentialités humaines, se retrouve dans une statis-

3 Walt Whitman Rostow, The Stages of Economic Growth, A Non-Communist Manifesto, Cambridge, Cambridge University Press, 1960.

4 Données tirées du site officiel du PNUD : http://hdrstats.undp.org/fr/indicateurs/49806.html, consulté en janvier 2011. 
tique récente établie à partir de la répartition hommes/femmes dans les conseils d'administration.

Tableau 1:

Proportion de femmes dans les CA des 300 plus grandes entreprises en $\%{ }^{5}$

\begin{tabular}{|r|r|}
\hline Norvège & 44,2 \\
\hline Suède & 26,9 \\
\hline Finlande & 25,7 \\
\hline Danemark & 18,0 \\
\hline Pays-Bas & 12,3 \\
\hline Royaume-Uni & 11,5 \\
\hline Irlande & 10,1 \\
\hline Autriche & 9,2 \\
\hline Allemagne & 7,8 \\
\hline France & 7,6 \\
\hline
\end{tabular}

Si l'excellent chiffre de la Norvège peut s'expliquer par une loi qui oblige les entreprises à avoir au moins $40 \%$ de femmes dans leur CA, les données suédoises, finlandaises et danoises ne doivent rien à de tels dispositifs. Enfin, les pays scandinaves triomphent également dans le domaine de l'éducation depuis des décennies, la Finlande obtenant régulièrement les meilleurs résultats dans les tests PISA de l'Organisation de coopération et de développement économique (OCDE) entre 2000 et $2009^{6}$.

Or, justement, s'il existe des pays où l'art de la prévision économique est pratiqué depuis le XVIII siècle, ce sont bien les pays scandinaves. Ainsi, la Suède est le premier royaume européen, et probablement du monde, à se doter d'un office national de statistiques, dès 1732 : le Tabellverket ${ }^{7}$. Cet organisme cherche à

5 Source : European Professional Women's Network (année 2008), http://www. europeanpwn.net/, site consulté en janvier 2011.

6 Site officiel de l'OCDE pour le programme PISA (Programme international de l'OCDE pour le suivi des acquis des élèves) : http://www.pisa.oecd.org/ dataoecd/33/5/46624382.pdf, site consulté en janvier 2011.

7 Ulf Jorner (dir.), Svensk jordbruksstatistik 200 år, Stockholm, SCB, 1999. 
anticiper les insuffisances de la production agricole et surveille l'évolution de la population. Il élabore des statistiques de plus en plus précises au fil du XIX $\mathrm{X}^{\mathrm{e}}$ siècle, notamment sur l'industrie et l'artisanat à partir de $1830^{\circ}$. Cette période correspond au règne de Jean-Baptiste Bernadotte, maréchal de Napoléon, devenu prince héritier puis roi de Suède et de Norvège sous le nom de Karl Johan, ou Charles XIV Jean, entre 1810 et $1844^{9}$. Une ère de paix de deux siècles débute alors et le gouvernement suédois s'appuie sur les données fournies par ses statisticiens afin d'adapter son économie à la concurrence croissante. En effet, l'économie suédoise s'avère très ouverte sur le monde par nécessité, elle importe de plus en plus de céréales, vu son climat, et exporte de plus en plus d'objets manufacturés en raison de l'étroitesse de son marché intérieur ${ }^{10}$.

La Suède essaye donc d'anticiper les difficultés et d'atténuer les crises en appliquant une politique économique pragmatique fondée sur des objectifs à long terme ${ }^{11}$. Dans un premier temps, les réserves d'or et d'argent sont reconstituées afin de retrouver une monnaie solide le plus rapidement possible après les coûteuses guerres du XVIII e siècle. Le retour à l'étalon argent s'effectue en 1834 après des années de limitation drastique des dépenses, en particulier dans le cadre de l'apparat royal, Charles XIV Jean, alias Bernadotte, inaugure de la sorte une tradition de monarchie peu dépensière et proche de ses sujets. Les moyens de transport -route, chemin de fer et Göta Kanal - bénéficient de toute l'attention de la nouvelle dynastie dans l'esprit de la philosophie saint-simonienne. L'exploitation des ressources naturelles - forêt,

8 Carl-Johan Gadd, "Präster och landshöwingar rapporterar. Allmän jordbruksstatistik 1802-1864 ", dans Ulf Jorner (dir.), Svensk jordbruksstatistik 200 år, Stockholm, SCB, 1999, p. 28-49.

9 Jean-Marc Olivier, «Bernadotte Revisited, or the Complexity of a Long Reign (1810-1844) ", Revue d'histoire nordique/Nordic Historical Review, $\mathrm{n}^{\circ}$ 2, octobre 2006, p. 127-137.

10 Lars Magnusson, An Economic History of Sweden, London/New York, Routledge, 2000.

11 Jean-Marc Olivier, "La politique économique de Charles XIV Jean de Suède-Norvège (1810-1844) : entre pragmatisme et vision à long terme ", Revue d'histoire nordique/Nordic Historical Review, $\mathrm{n}^{\text {os }} 6 / 7$, juin 2009, p. 164174. 
minerai de fer et énergie hydraulique - se rationalise au fil du $\mathrm{XIX}^{\mathrm{e}}$ siècle grâce à cet essor des infrastructures de communication. Enfin, les dirigeants suédois se persuadent de l'intérêt d'exporter des objets à plus haute valeur ajoutée afin de créer des emplois et de la richesse sur leur sol. Ils le font en occupant des niches d'activités complémentaires de la puissante industrie anglaise de la première moitié du XIX ${ }^{\mathrm{e}}$ siècle. Les aciers spéciaux offrent alors de belles opportunités dans les domaines de l'outillage, des machines et des armes, aboutissant à la naissance de grands groupes comme SKF, spécialisé dans les roulements à billes, puis Volvo. Ce souci d'émancipation et de prospérité transparaît régulièrement dans les écrits du roi Charles XIV Jean qui ne peut fonder sa nouvelle dynastie, très fragilisée en 1815 , que sur le soutien de son peuple ${ }^{12}$.

La volonté de préparer l'avenir, au moins de manière macroéconomique, s'affirme au $\mathrm{XX}^{\mathrm{e}}$ siècle avec l'arrivée des sociauxdémocrates au pouvoir à partir des années 1920. Gunnar et Alva Myrdal élaborent le concept " d'harmonie créée » ou " construite » par opposition à l'harmonie spontanée de la main invisible ${ }^{13}$. Pour cela ils s'appuient sur leurs idéaux socialistes, mais aussi sur la tradition nordique du Samhälle suédois ou du Samfunn norvégien, termes qui désignent la communauté de valeurs devant laquelle les individus doivent s'incliner. Cette notion s'enracine loin dans l'histoire sociale scandinave car elle fait référence aux

$\overline{12}$ Les archives de Charles XIV Jean, ou archives Bernadotte, comportent 265 cartons conservés au palais royal de Kungliga Slottet à Stockholm et peuvent être transférées, après autorisation royale, aux Riksarkivet (archives nationales) pour consultation. Les cotes exactes sont : "Bernadotteska Familjearkivet (BFA) - Karl XIV Johans arkiv - Nr 1-265", signifiant "Archives de la famille Bernadotte, archives de Karl XIV Johan, cotes 1 à 265 ». Il existe un guide en suédois de l'ensemble du fonds : Ingemar Carlsson, Kungens Familjearkiv, Stockholm, Carlsson Bokförlag, 1994. Voir également : Jean-Marc Olivier, "Les archives de Bernadotte devenu Charles XIV Jean de Suède-Norvège (1792-1844) ", Revue d'histoire nordique/Nordic Historical Review, $\mathrm{n}^{\circ} 2$, octobre 2006, p. 203-213. Ces archives présentent le grand avantage d'être pour l'essentiel en français car Jean-Baptiste Bernadotte n'a jamais réussi à apprendre le suédois.

13 Gilles Dostaler, Diane Éthier et Laurent Lepage, Gunnar Myrdal et son œuvre, Paris, Economica, 1990, 230 p. 
vikings, aux solidarités hivernales rurales, aux marins dont il faut secourir les veuves, à la communauté luthérienne qui élit ses pasteurs etc.

Gunnar Myrdal, chargé de la construction d'un État providence total à partir du début des années 1940, recourt à l'appareil statistique suédois de plus en plus perfectionné et permettant d'effectuer des projections ${ }^{14}$. Il mobilise les démographes qui calculent l'accroissement prévisible de la population, mais aussi son vieillissement et l'évolution de son degré de qualification. Les experts de la finance établissent le niveau du prélèvement fiscal nécessaire au bon fonctionnement du Welfare State. Enfin, les économistes coordonnent l'ensemble. Gunnar Myrdal obtient le prix Nobel d'économie dès 1974 pour cette œuvre, avant sa femme Alva en 1982.

Cette vaste entreprise de développement équilibré, protégeant le citoyen du berceau jusqu'à la tombe tout en préservant le dynamisme du capitalisme, aboutit dès les années 1950. À cette date, une assurance maladie généralisée fonctionne, l'école unique est obligatoire pendant neuf ans et le système de pensions de retraite offre une véritable autonomie à toutes les personnes âgées. Surtout, l'État providence suédois se révèle capable de se réformer par anticipation. Ainsi, prenant en compte l'allongement de l'espérance de vie, le gouvernement enclenche une réflexion nationale dès les années 1980 sur la réforme du système de retraite, aboutissant à des modifications efficaces et consensuelles.

Au total, même si certains calculs financiers se sont révélés approximatifs, les mécanismes de prévision ont globalement bien fonctionné et, dès 1961, la Suède et la Norvège sont présentées comme les deux pays disposant du meilleur système de protection

$\overline{14}$ Depuis 1870 paraît chaque année un recueil de tableaux présentant les principales données de la statistique officielle, mais il ne s'agit que de résumés (Sveriges officiella statistik $i$ sammandrag, le premier volume publié en 1870 compte 68 pages). À partir de 1914, un véritable annuaire statistique est publié (ex : Statistisk årsbok för Sverige. Första Argangen,1914 [Annuaire statistique de la Suède. $1^{\mathrm{re}}$ année, 1914], Stockholm, Bureau central de statistique, 1914). 
sociale au monde. Cette obsession de préparer l'avenir se retrouve aujourd'hui en Norvège avec le « fonds pétrole » destiné à assurer la perpétuation d'un niveau de vie élevé au-delà de l'épuisement des ressources pétrolières en mer du Nord. Ainsi, seul les intérêts des revenus tirés du pétrole sont utilisés tandis que l'essentiel des capitaux est placé à long terme ${ }^{15}$. Les enseignements de cette réussite économique scandinave et suisse peuvent se résumer à une précaution sage : à défaut de pouvoir prédire l'avenir, il faut anticiper les difficultés qu'il promet.

\section{Bibliographie}

Bergier, Jean-François, Histoire économique de la Suisse, Paris, Armand Colin, 1984.

Carlsson, Ingemar, Kungens Familjearkiv, Stockholm, Carlsson Bokförlag, 1994.

Carrez, Maurice et Jean-Marc Olivier, "Réflexion historiographique sur l'industrialisation des pays scandinaves ", Revue d'histoire nordique, $\mathrm{n}^{\circ} 1$, décembre 2005, p. 16-22.

Dostaler, Gilles, Diane Éthier et Laurent Lepage, Gunnar Myrdal et son cuvre, Paris, Economica, 1990.

Gadd, Carl-Johan, « Präster och landshöwingar rapporterar. Allmän jordbruksstatistik 1802-1864 ", dans Ulf Jorner (dir.), Svensk jordbruksstatistik 200 år, Stockholm, SCB, 1999, p. 28-49.

Grasland, Claude, " 25 ans de développement humain ", dans Images économiques du monde, Paris, Armand Colin, 2005, p. 43-54.

Jorner, Ulf (dir.), Svensk jordbruksstatistik 200 år, Stockholm, SCB, 1999.

Magnusson, Lars, An Economic History of Sweden, London/New York, Routledge, 2000.

Olivier, Jean-Marc, " Aux origines du miracle suédois », L'Histoire, n 354, juin 2010, p. 66-73.

15 Jean-Marc Olivier, « La Norvège deuxième actionnaire du CAC 40! Le succès économique de ce petit pays ne s'explique pas seulement par ses hydrocarbures ", Le Monde, mercredi 31 décembre 2008, p. 15. 
Olivier, Jean-Marc, "La politique économique de Charles XIV Jean de Suède-Norvège (1810-1844) : entre pragmatisme et vision à long terme ", Revue d'histoire nordique/Nordic Historical Review, n ${ }^{\text {os }}$ 6/7, juin 2009, p. 164-174.

Olivier, Jean-Marc, "La Norvège deuxième actionnaire du CAC 40! Le succès économique de ce petit pays ne s'explique pas seulement par ses hydrocarbures ", Le Monde, mercredi 31 décembre 2008, p. 15.

Olivier, Jean-Marc, "Bernadotte Revisited, or the Complexity of a Long Reign (1810-1844) ", Revue d'histoire nordique/Nordic Historical Review, $\mathrm{n}^{\circ}$ 2, octobre 2006, p. 127-137.

Olivier, Jean-Marc, "Les archives de Bernadotte devenu Charles XIV Jean de Suède-Norvège (1792-1844) ", Revue d'histoire nordique/Nordic Historical Review, $\mathrm{n}^{\circ}$ 2, octobre 2006, p. 203-213.

Olivier, Jean-Marc, "L'industrialisation rurale douce de l'arc jurassien franco-suisse : un modèle montagnard? ", Ruralia, $\mathrm{n}^{\circ}$ 4, 1999, p. 11-22.

Rostow, Walt Whitman, The Stages of Economic Growth, A Non-Communist Manifesto, Cambridge, Cambridge University Press, 1960.

Statistisk årsbok för Sverige. Första Argangen,1914 [Annuaire statistique de la Suède. 1'e année, 1914], Stockholm, Bureau central de statistique, 1914). 